\title{
The role of loss of control and predictability in the hypoalgesic response to noncontingent food delivery in rats
}

\author{
D. M. HODGSON and N. W. BOND \\ Macquarie University, Sydney, New South Wales., Australia
}

\begin{abstract}
Exposure to noncontingent food delivery has been shown to elicit an increase in nociceptive thresholds in food-deprived rats. In Experiment 1, we examined whether this hypoalgesic response might be attributed to a loss of control over food delivery. Rats were exposed to noncontingent food delivery with or without prior exposure to contingent food delivery, and nociceptive thresholds were recorded using the tail-immersion test. Both groups of rats exhibited significant increases in nociceptive thresholds, and no differences were found between rats that had experienced a loss of control and those that had not. In Experiment 2, we examined the role of predictability in the hypoalgesic response to noncontingent food delivery. Food-deprived rats were exposed to noncontingent food delivery with or without a light signal preceding the delivery of each food pellet. Both groups of animals exhibited significant increases in nociceptive thresholds. However, the hypoalgesic response was significantly reduced when food delivery was preceded by a light signal. These findings suggest that it is not merely a lack of control over food delivery which may be responsible for the activation of analgesic mechanism, but that the availability of predictability has an important modulatory role as well.
\end{abstract}

Exposure to a wide variety of stressors has been shown to increase nociceptive thresholds in rats. This phenomenon, referred to as "stress-induced analgesia" (SIA), involves the activation of multiple pain inhibitory systems - some opioid mediated, others nonopioid mediated-that inhibit nociception via neural and/or hormonal mechanisms (for reviews, see Bodnar, 1986; Kelly, 1986; Rodgers \& Randall, 1988). Although much of the research on SIA has utilized nociceptive stimuli, there is evidence that nonnociceptive stimuli can also activate these mechanisms (Amir \& Amit, 1978; Bodnar, Kelly, Spiaggia, \& Glusman, 1977; Hayes, Bennett, Newlon, \& Mayer, 1978). Food deprivation, for example, has been found to increase nociceptive thresholds in rats (Biles, Illich, \& Grau, 1992; Bodnar, Kelly, Spiaggia, \& Glusman, 1978; Hamm, Knisely, Watson, Lyeth, \& Bossut, 1985; McGivern, Berka, Berntson, Walker, \& Sandman, 1979). The hypoalgesia elicited by $24 \mathrm{~h}$ of food deprivation is classified as opioid/hormonal given that it is attenuated by hypophysectomy, adrenalectomy, and naloxone administration (Hamm et al., 1985).

There have been a number of different proposals concerning the factors that are critical in determining the na-

\footnotetext{
The authors state that all treatments and procedures used in this research project were carried out in accordance with APA ethical standards. All procedures were approved by the Macquarie University Animal Ethics Committee. The authors would like to thank Wayne McTegg for animal maintenance and Len Glue for assistance with equipment. The authors' mailing address is School of Behavioural Sciences, Macquarie University, Sydney, NSW 2109, Australia.
}

ture of the hypoalgesic response. Most of these proposals focus on the physical characteristics of the stressor, such as the intermittency of shock (Lewis, Cannon, \& Liebeskind, 1980), the severity of shock (Terman, Shavit, Lewis, Cannon, \& Liebeskind, 1984), or the body region shocked (Watkins, Cobelli, Faris, Aceto, \& Mayer, 1982). However, psychological aspects of the stressor have also been found to be important. Maier and colleagues have demonstrated that shock controllability has an important modulatory role in SIA (Maier, 1986). Jackson, Maier, and Coon (1979) demonstrated that rats exposed to 80 inescapable shocks display an increase in nociceptive thresholds upon brief reexposure to shock $24 \mathrm{~h}$ later. Exposure to the same number of escapable shocks does not produce this "long-term" analgesia, and only the analgesia elicited by inescapable shock is reversible by naloxone (Maier et al., 1980).

Evidence is now emerging that controllability of a nonnociceptive stimulus also activates endogenous pain inhibitory systems. Tazi, Dantzer, and Le Moal (1987) examined the effect of control over food delivery on nociceptive thresholds, and reported that food-deprived rats unable to control the delivery of food (i.e., noncontingent food delivery) had significantly higher tail-flick latencies than did rats who were able to control the delivery of food (i.e., contingent food delivery). Subsequent research has extended these original findings by demonstrating that the response to noncontingent, but not contingent, food delivery is mediated by opioid mechanisms (Hodgson \& Bond, 1991). The authors suggest that it is the lack of control over food delivery that is re- 
sponsible for the activation of opioid pain-inhibitory mechanisms. The present research, however, considers two alternative explanations. The first experiment addresses the possibility that the hypoalgesic response to noncontingent food delivery is due to the loss of control, rather than to the lack of control, over food delivery. The second experiment examines the possibility that the hypoalgesic response to noncontingent food delivery may be attributed to the lack of predictability of food delivery.

\section{EXPERIMENT 1}

Hodgson and Bond (1991) have suggested that it is the lack of control over food delivery that is responsible for the hypoalgesic response to noncontingent food delivery. However, the protocol used in their research confounds the effects of lack of control with those of loss of control. In their preliminary training phase, animals are exposed to contingent (i.e., controllable) food delivery prior to noncontingent (i.e., uncontrollable) food delivery. According to several lines of evidence, this change in reinforcement contingency (i.e., loss of control) can be more aversive to the organism than a lack of control. For example, rhesus monkeys exhibit severe stress symptomatology when a lever that previously controlled a noxious stimulus is removed (Stroebel, 1969). Weiss (1971) found that animals that experience a reduction in the effectiveness of an escape response show greater ulceration in response to shock than do animals who have never had control over shock. Similarly, animals experiencing a loss of control show more dramatic increases in corticosterone and norepinephrine than do animals who have no control (Coover, Ursin, \& Levine, 1973; Hanson, Larson, \& Snowdon, 1976; Mason, Brady, \& Tolson, 1966). The effect of loss of control has also been reported in the appetitive paradigm. Rats and pigeons exposed to noncontingent food delivery following a prior history of contingent food delivery exhibit impaired escape responding (Goodkin, 1976; Sonada, Okayasu, \& Hirai, 1991). Escape performance is significantly more impaired in rats that experience a loss of control as opposed to a lack of control (Sonada, et al., 1991).

Given the above findings, this experiment examines the possibility that the hypoalgesic response to noncontingent food delivery may be attributed to the impact of loss of control. Loss of control is operationalized as the change from contingent to noncontingent reinforcement. Experiment 1 examined the hypoalgesic responses of animals exposed to noncontingent food delivery with or without prior exposure to contingent food delivery.

\section{Method}

Subjects. Thirty-two male Wistar rats, 60 days old, were obtained from the Australian Resource Centre, Western Australia. The subjects were 80 days old and weighed approximately $370 \mathrm{~g}$ at the start of the experiment. They were housed individually in cages measuring $42 \times 28 \times 15 \mathrm{~cm}$ in a single colony room on a 12-h light/dark cycle (lights on $0700 \mathrm{~h}$ ).

Apparatus. Testing was carried out in three identical operant chambers $(20 \times 24 \times 28 \mathrm{~cm})$, each housed in a sound-attenuating box $(50 \times 60 \times 70 \mathrm{~cm})$. Food pellets weighing $45 \mathrm{mg}$ (P. J. Noyes Co.) could be delivered into a food tray recessed at the base of the chamber.

Nociceptive testing. Prior to and following each test session, each rat was tested for pain responsiveness using the tail-immersion test (Jannsen, Niemegers, \& Dony, 1963). In this test, the lower $3 \mathrm{~cm}$ of the tail is marked. Testing involves wrapping the animal firmly but gently in a toweling cloth while the lower $3 \mathrm{~cm}$ of the tail is immersed in water, maintained at $52^{\circ} \mathrm{C}$, until the typical withdrawal response is observed. Tail-flick latencies were measured manually using a stopwatch accurate to $.01 \mathrm{sec}$, and maximum immersion time was limited to $15 \mathrm{sec}$. Animals were familiarized with the cloth restraint method prior to the experiment proper.

Procedure. All rats were handled daily for 3 weeks. They were housed individually and food and water were available ad lib. After this period, all animals were food deprived for $48 \mathrm{~h}$ and randomly allocated to one of three groups $(n=8)$, Groups noncontingent $/+$, noncontingent $/-$, and $E C$ (experimental control). All subjects then underwent two preliminary training sessions. On Day 1 of this procedure, rats in the noncontingent groups were placed individually in an operant chamber for 15 min with 30 pellets in the food tray. On the following day, each rat was again placed in the operant chamber for $15 \mathrm{~min}$, but this time 30 food pellets were delivered individually at $30-\mathrm{sec}$ intervals. Rats in the $\mathrm{EC}$ group also underwent the same two preliminary training sessions. However, during these sessions each rat was placed in the operant box for $15 \mathrm{~min}$ with 30 food pellets freely available in the food tray. All animals were then given a further four training sessions, each of $15 \mathrm{~min}$ duration. During these sessions, animals in the noncontingent $/+$ group were shaped to leverpress using a continuous reinforcement schedule, animals in the noncontingent - group were not shaped to leverpress but received 30 food pellets according to a fixed time schedule ( $30 \mathrm{sec})$ during each of the training sessions. Animals in the EC group were placed in the operant box for the same length of time as rats in the noncontingent groups, with 30 food pellets available in the food tray, but were not shaped to leverpress.

Following the preliminary training, the subjects were deprived of food for $24 \mathrm{~h}$, and then submitted to five daily 15 -min test sessions. During the test sessions, rats in the noncontingent groups received 30 food pellets according to a fixed time $(30-\mathrm{sec})$ schedule. Rats in the EC group were placed in the operant box with 30

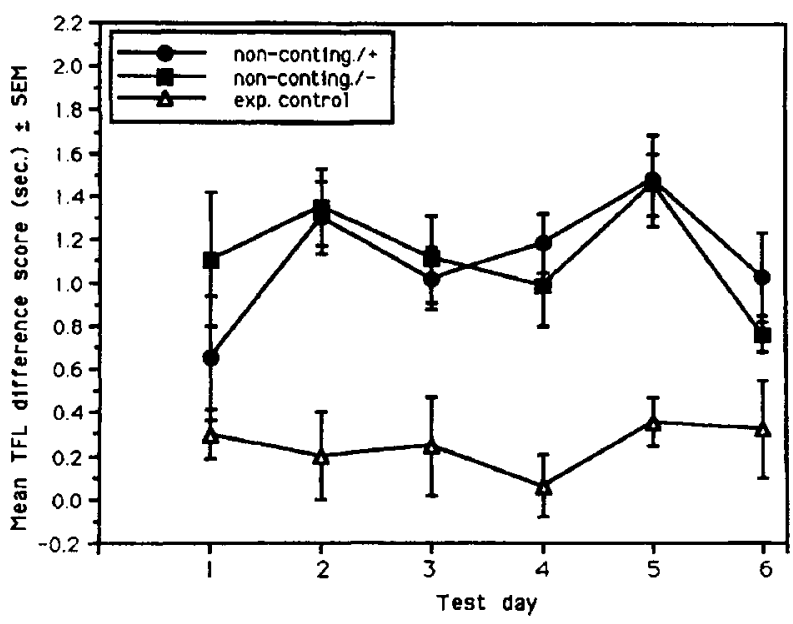

Figure 1. Mean tail-flick latency (TFL) difference scores (postpretest) for animals exposed to noncontingent food delivery with (i.e., non-conting. $/+$ ) or without (i.e., non-conting. $/-$ ) prior exposure to contingent food delivery, and for animals in the experimental control group (i.e., exp. control). 
pellets available in the food tray. Measures of nociception were obtained using the tail-immersion test prior to and immediately after the 15-min test session. Testing was carried out from 0600 to $0900 \mathrm{~h}$. The animals were maintained at $80 \%$ of their ad-lib body weights for the duration of the experiment.

\section{Results}

As illustrated in Figure 1, there appears to be little difference in tail-flick latencies between animals exposed to noncontingent food delivery with or without prior exposure to contingent food delivery on most of the test days. On Day 1, however, there is some evidence to suggest that prior exposure to controllable food delivery may have attenuated the hypoalgesic response to noncontingent food delivery. On Test Day 1, the difference between pre- and posttest tail-flick latencies is reduced in animals with prior exposure to contingent food delivery.

Data analysis utilizing a two-way repeated measures analysis of variance (ANOVA), group (3) $\times$ day (6) on difference scores (post-pretest latencies), revealed significant group $[F(2,21)=22.61, p<.001]$ and day effects $[F(5,105)=2.61, p=.029]$. The interaction between group and day was not significant $[F(10,105)=$ $1.237, p=.28]$. Multiple comparisons (Bonferroni) revealed that the difference between pre- and posttest latencies was significantly greater in the noncontingent/ + $[t(21)=5.78, p<.001]$ and noncontingent $/-$ groups $[t(21)=5.92, p<.001]$ than in the EC group. The difference between the noncontingent $/+$ and noncontingent/ - groups was not significant. Although the day effect was also found to be significant, multiple comparisons revealed no significant differences. The comparison between Day 1 and Day 5 is approaching significance $[t(105)=2.90, p=.066]$, with the difference between pre- and posttest latencies being greater on Day 5 . The lack of significant differences between days is most likely due to the reduction in alpha level required to control for Type I error.

A second analysis was carried out to investigate whether the reduction in difference scores noted in the noncontingent/+ group on Day 1 was significant. A one-way ANOVA on difference scores from Test Day 1 revealed, however, that the group effect was not significant $[F(2,21)=2.59, p=.10]$.

\section{Discussion}

The present study demonstrates that noncontingent food delivery elicits a significant increase in nociceptive thresholds. It was hypothesized that the hypoalgesic response to noncontingent food delivery might be due to the loss of control brought about by the change in reinforcement contingency. This hypothesis is not supported given that an equivalent increase in nociceptive sensitivity was observed in animals exposed to noncontingent food delivery with or without prior experience with contingent food delivery. There was some evidence to suggest that prior exposure to contingent food delivery might have had a transient "immunizing" effect, although data analysis limited to Test Day 1 revealed that this effect was not significant. At this point, therefore, it remains tenable that it is the uncontrollability of food delivery that is responsible for the activation of analgesic mechanisms. However, an important consideration for any study examining the effects of controllability is the possibility that controllability is confounded with predictability. Experiment 2 examined the possibility that the hypoalgesic response to noncontingent food delivery might be accounted for by the unpredictability of food delivery.

\section{EXPERIMENT 2}

The availability of control is known to mediate the behavioral and physiological response of an organism to stress. (Davis et al., 1977; Dess, Linwick, Patterson, Overmier, \& Levine, 1983; Weiss, 1971). However, there is also evidence to suggest that many of the effects previously attributed to a lack of control may in fact be due to a lack of predictability (Mineka, Cook, \& Miller, 1984; Mineka \& Henderson, 1985). Behavioral and physiological consequences of unpredictable shock are similar to those of uncontrollable shock, resulting in greater pituitary-adrenal activation (Dess et al., 1983), more severe gastric ulceration (Weiss, 1971), and decreased responsiveness to nociceptive stimuli (Guile \& McCutcheon, 1984) in comparison with predictable shock. Unpredictable shock also impairs appetitive instrumental responding (Seligman, 1968), and rats exhibit a preference for predictable over unpredictable food delivery (Prokasy, 1956).

If the effects of controllability are reducible to those of predictability, increasing predictability should function in the same manner as increasing control. Evidence supporting this proposal is provided by the finding that exposure to uncontrollable shock produces higher levels of fear than exposure to controllable shock. The provision of a feedback stimulus (i.e., signal after shock) attenuates the fear response to the same level as that exhibited by animals who have control over shock (Mineka et al., 1984). Furthermore, provision of a feedback signal eliminates the interference effects observed after exposure to uncontrollable shock (Volpicelli, Ulm, \& Altenor, 1984). According to these findings the effects of control appear to be reducible to those of predictability. The feedback stimulus signals shock termination and a safety period, while the absence of a signal has behavioral and physiological consequences for the organism because of the unpredictability of shock absence.

The question of interest is whether the hypoalgesic response to noncontingent (i.e., uncontrollable) food delivery is a consequence of the unpredictability of food delivery. If this is the case, provision of a signal predicting food delivery should attenuate the hypoalgesic response. This experiment examines the hypoalgesic response of animals exposed to noncontingent food delivery with or without a signal preceding food delivery. 


\section{Method}

Subjects. The subjects were 32 naive male Wistar rats, 80 days old and weighing approximately $370 \mathrm{~g}$ at the start of the experiment. They were housed individually as outlined in Experiment 1.

Apparatus. The apparatus was as outlined in Experiment 1.

Nociceptive testing. Nociceptive testing was as outlined in Experiment 1 .

Procedure. All rats were handled daily for 3 weeks. They were housed individually and food and water were available ad lib. After this period, the rats were food deprived for $48 \mathrm{~h}$ and randomly allocated to one of four groups $(n=8)$, Groups noncontingent, noncontingent/light, EC (i.e., experimental control), and EC/light (i.e., experimental control/light). All subjects then underwent the preliminary training sessions outlined in Experiment 1. Animals in the noncontingent groups were then shaped to leverpress in four 15-min consecutive sessions. Animals in the two experimental control groups were placed in the operant box in each of the four sessions, for the same period of time as the test animals. They received 30 food pellets but were not shaped to leverpress.

Following the preliminary training sessions, the animals were food deprived for $24 \mathrm{~h}$ and then submitted to five daily 15 -min test sessions. During the test sessions, rats in the noncontingent/light group received 30 food pellets according to a variable time (30-sec) schedule, with a light preceding the delivery of each pellet by $2 \mathrm{sec}$. Animals in the noncontingent group received 30 food pellets according to the same reinforcement schedule (VT/30 sec) but without the light signal. Rats in the $\mathrm{EC}$ and $\mathrm{EC} /$ light groups were placed in the operant box for the same amount of time as animals in the noncontingent groups, with 30 pellets available in the food tray. For animals in the EC/light group, a light signal was delivered on a variable time schedule $(30 \mathrm{sec})$. Measures of nociception were obtained using the tail-immersion test prior to and immediately after the 15 -min test session. Testing was carried out from 0600 to $0900 \mathrm{~h}$. The rats were maintained at $80 \%$ of their adlib body weights for the duration of the experiment.

\section{Results}

As shown in Figure 2, noncontingent food delivery elicits an increase in tail-flick latencies whether food delivery is signaled or unsignaled. However, the hypoalgesic response is more pronounced when food delivery is not preceded by a light signal. As in Experiment 1 ,

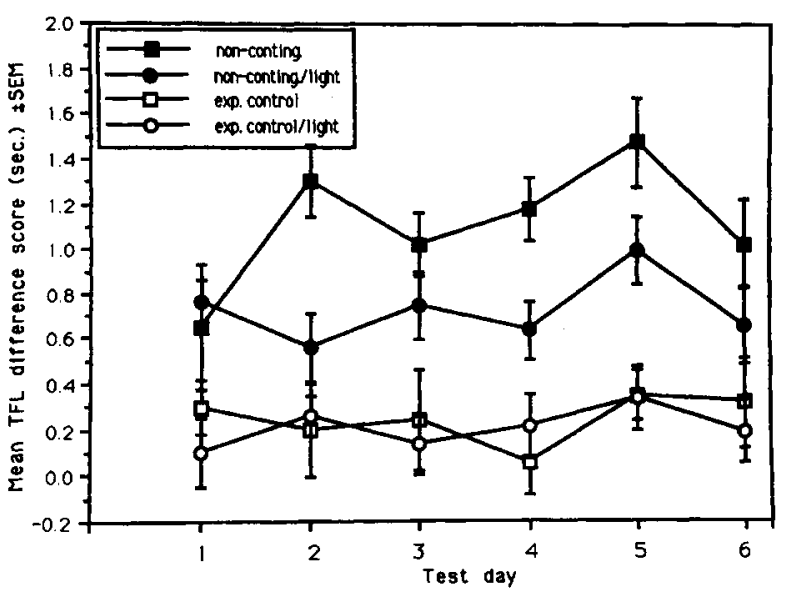

Figure 2. Mean tail-flick latency (TFL) difference scores (postpretest) for animals exposed to noncontingent food delivery with (i.e., non-conting./ light) or without (i.e., non-conting.) a light signal, and for animals in the experimental control groups with (i.e., exp. control/light) or without (i.e., exp. control) a light signal. tail-flick latency-change scores on Test Day 1 appear to be suppressed in animals exposed to noncontingent food delivery with prior exposure to contingent food delivery.

Data analysis utilizing a two-way repeated measures ANOVA, group $(2) \times$ light $(2) \times$ day (6) on difference scores (post-pretest latencies), resulted in a significant interaction between group and light $[F(1,28)=4.42, p<$ $.05]$. Multiple comparisons (Bonferroni) revealed that the difference between pre- and posttest latencies was significantly higher in the noncontingent group than in the noncontingent/light group $[t(28)=3.32, p<.01]$. The difference score for both the noncontingent and noncontingent/light groups were significantly higher than those in the EC or EC/light groups $[t(28)=11.8$, $p<.001$, and $t(28)=4.51, p<.001$, respectively]. The difference score in the EC group was not significantly different from that in the EC/light group.

An additional two-way ANOVA, group (2) $\times$ light (2), limited to Test Day 1 revealed a significant group effect $[F(1,28)=8.23, p=.01]$. The difference in tail-flick latencies was significantly higher in the noncontingent group than in the EC group. However, the interaction between group and light signal was not significant $[F(1,28)=$ $.77, p>.05]$, suggesting that the effect for group is not consistent with regard to the presence or absence of a light signal.

\section{Discussion}

The findings of this experiment demonstrate that noncontingent food delivery in food-deprived rats results in a significant increase in tail-flick latencies. Provision of a light signal prior to the delivery of each pellet significantly attenuates the hypoalgesic response. As such, the present findings suggest that predictability plays a role in the rats' response to noncontingent food delivery.

Hodgson and Bond (1991) have previously hypothesized that the hypoalgesic response to noncontingent food delivery is due to the aversive nature of uncontrollable food delivery. However, a continuing point of debate in the literature is the possibility that effects attributed to a lack of control may, in fact, be due to a lack of predictability. Control, for instance, may exert its effects by allowing the animal to predict an event. This proposal is supported by the finding that effects similar to those obtained when animals are given control over an event can be obtained by providing the animal with a feedback stimulus (i.e., signal after shock). Provision of a feedback signal will block the escape deficits (Volpicelli et al., 1984), context fear (Mineka et al., 1984), and opioid analgesia typically elicited by inescapable shock (Maier \& Keith, 1987; Maier \& Warren, 1988). A signal prior to shock has similar consequences in that it results in less severe stress symptomatology, such as gastric ulceration, weight loss, and corticosterone elevations (Tsuda, Ida, Satoh, Tsujimaru, \& Tanaka, 1989; Weiss, 1968, 1970), and attenuates fear conditioned to contextual cues (Maier \& Keith, 1987). However, in contrast to the findings using a feedback stimulus, a signal prior to shock has no ef- 
fect on escape deficits or hypoalgesia (Maier \& Keith, 1987).

In the appetitive paradigm, a lack of predictability has also been shown to have effects similar to those associated with a lack of control. Pigeons show retarded learning following unsignaled food delivery (Tomie, Murphy, \& Faith, 1980; Wasserman \& Molina, 1975), and rats exhibit a preference for predictable over unpredictable food delivery (Prokasy, 1956). Unpredictable food delivery has also been shown to elicit a more pronounced hypoalgesia than predictable food delivery (Tazi et al., 1987). In accordance with these studies, the present study found that the provision of a predictive signal (i.e., prior to food delivery) attenuates the hypoalgesic response to noncontingent food delivery. These findings, however, are in contrast to those observed in the shock paradigm, where a signal after shock termination, but not before, attenuates the hypoalgesic response to shock (Maier \& Keith, 1987). It is possible that these differences are due to the fact that a signal prior to shock predicts pain, and a signal after shock predicts a pain-free period. It would appear logical, therefore, that hypoalgesia would be attenuated only when a pain-free period was predicted. In the appetitive situation, a signal prior to food signals reinforcement, while a signal after food signals its discontinuation. Again, it would seem logical that hypoalgesia would not be attenuated when a signal predicted an aversive event such as the discontinuation of reinforcement. This remains to be investigated in future research.

\section{GENERAL DISCUSSION}

The findings presented in this paper demonstrate that exposure to noncontingent food delivery in food-deprived animals elicits an increase in nociceptive thresholds. Hodgson and Bond (1991) suggest that it is the lack of control over food delivery that is responsible for the activation of analgesic mechanisms. Experiment 1 examined the possibility that the hypoalgesic response might be attributed more specifically to a loss of control rather than a lack of control. This, however, was not found to be the case. Animals exposed to a loss of control over food delivery exhibited a hypoalgesic response that was equivalent to that exhibited by animals exposed to food delivery that was merely uncontrollable.

Experiment 2 addressed the possibility that the hypoalgesic response, attributed to a lack of control over food delivery, might in fact be attributed to a lack of predictability over food delivery. Control may be important only because of the predictability it provides the animal. Some support for this proposal was provided by the finding that increasing predictability via the provision of a light signal attenuated the hypoalgesic response to noncontingent food delivery. The fact that the hypoalgesia response was only partially attenuated may be accounted for by one of two possible explanations. First, it is possible that the light signal did not function sufficiently as a salient cue, and this remains to be investigated in future research. Second, and more importantly, it suggests that in this instance prediction and control are not reducible to one factor, but are instead independent variables that interact.

Weiss (1971) suggests that the effects of predictability and controllability are, in fact, additive. In a study on the effects of shock-induced gastric ulceration, Weiss demonstrated that the absence of a warning signal added to the large effect produced by uncontrollable shock. The addition of a signal decreased the incidence of ulceration. Weiss subsequently predicted that the highest incidence of stress pathology was a result of unpredictable/ uncontrollable events, that the lowest incidence was a result of controllable/predictable events, and that intermediate levels occur in response to uncontrollable/predictable and controllable/predictable events. In line with this proposal, Experiment 2 found that the hypoalgesic response was more marked when food delivery was unpredictable/uncontrollable than when food delivery was uncontrollable/predictable. In light of these findings, it would be of interest to consider a more detailed examination of the applicability of Weiss's additive model to the current appetitive paradigm.

\section{REFERENCES}

AmIr, S., \& AmIт, Z. (1978). Endogenous opioid ligands may mediate stress-induced changes in the affective properties of pain-related behaviour in rats. Life Sciences, 23, 1143-1152.

Biles, M. K., Illich, P. A., \& Grau, J. W. (1992). Long-term effects of food deprivation: II. Impact on morphine reactivity. Psychobiology, 20, 85-91.

BODNAR, R. J. (1986). Neuropharmacological and neuroendocrine substrates of stress-induced analgesia. In D. D. Kelly (Ed.), Stress induced analgesia (Annals of the New York Academy of Sciences, Vol. 467, pp. 345-361). New York: New York Academy of Sciences

Bodnar, R. J., Kelly, D. D., Spiaggia, A., \& Glusman, M. (1977). Analgesia produced by cold-water stress: Effect of naloxone. Federation Proceedings, 36, 1010.

Bodnar, R. J., Kelly, D. D., Spiaggia, A., \& Glusman, M. (1978). Biphasic alterations of nociceptive thresholds induced by food deprivation. Physiological Psychology, 6, 391-395.

Coover, G., Ursin, H., \& Levine, S. (1973). Plasma corticosterone levels during active avoidance learning in rats. Journal of Comparative \& Physiological Psychology, 82, 170-174.

Davis, H., Porter, J., Livingston, J., Herrman, T., MacFadden, J., \& Levine, S. (1977). Pituitary-adrenal activity and leverpress shock-escape behavior. Physiological Psychology, 5, 280-284.

Dess, N. K., Linwick, D., Patterson, J., Overmier, J. B., \& Levine, S (1983). Immediate and proactive effects of controllability and predictability on plasma cortisol responses to shock in dogs. Behavioral Neuroscience, 97, 1005-1016.

GoodKIN, F. (1976). Rats learn the relationship between responding and environmental events. An expansion of the learned helplessness hypothesis. Learning \& Motivation, 7, 382-393.

GuILE, M. N., \& McCuTcheon, N. B. (1984). Effects of naltrexone and signalling inescapable shock on nociception and gastric lesions in rats. Behavioral Neuroscience, 98, 695-702.

HaMm, R. J., KNisely, J. S., Watson, A., Lyeth, B. G., \& Bossut, D. F. B. (1985). Hormonal mediation of the analgesia produced by food deprivation. Physiology \& Behavior, 35, 879-882.

Hanson, J. D., Larson, M. E., \& Snowdon, C. T. (1976). The effects of control over high intensity noise on plasma cortisol levels in rhesus monkeys. Behavioral Biology, 16, 333-340.

Hayes, R. L., Bennett, G. J., Newlon, P. G., \& Mayer, D. J. (1978). 
Behavioral and physiological studies of non-narcotic analgesia in the rat elicited by certain environmental stimuli. Brain Research, $155,69-90$.

Hodgson, D. M., \& Bond, N. W. (1991). Control of food delivery in food deprived rats mediates analgesia. Behavioral Brain Research, 44, 205-209.

JACKSON, R. L., MAIER, S. F., \& CoON, D. J. (1979). Long term analgesic effects on inescapable shock and learned helplessness. Science, 206, 91-93.

JansSEN, P. A. J., Niemegeers, C. J. E., \& Dony, J. G. H. (1963). The inhibitory effect of fentanyl and other morphine-like analgesics on the warm water-induced tail withdrawal reflex in rats. Arzneimittel Forschung: Drug Research, 13, 502-507.

KELLY, D. D. (Ed.) (1986). Stress-induced analgesia (Annals of the New York Academy of Sciences, Vol. 467). New York: New York Academy of Sciences.

Lewis, J. W., CANNON , J. T., \& Liebeskind, J. C. (1980). Opioid and nonopioid mechanisms of stress analgesia. Science, 208, 623-625.

MAIER, S. F. (1986). Stressor controllability. In D. D. Kelly (Ed.), Stress-induced analgesia (Annals of the New York Academy of Sciences, Vol. 467, pp. 55-72). New York: New York Academy of Sciences.

Maier, S. F., Davies, S., Grau, J. W., Jackson, R. L., Morrison, D. H., MoYe, T., Madden, J., \& Barchas, J. D. (1980). Opiate antagonists and long-term analgesic reaction induced by inescapable shock in rats. Journal of Comparative \& Physiological Psychology, 94, 1172-1183.

MAIER, S. F., \& KeITh, J. R. (1987). Shock signals and the development of stress-induced analgesia. Journal of Experimental Psychology: Animal Behavior Processes, 13, 226-238.

MAIER, S. F., \& WARREN, D. A. (1988). Controllability and safety signals exert dissimilar proactive effects on nociception and escape performance. Journal of Experimental Psychology, 14, 18-25.

MASON, J. W., BRADY, J. V., \& Tolson, W. W. (1966). Behavioral adaptations and endocrine activity: Psychoendocrine differentiation of emotional states. In R. Levine (Ed.), Endocrines and the central nervous system. Baltimore: Williams \& Wilkins.

MCGivern, R., BERKa, C., BernTSON, G. G., WALKER, J. M., \& SANDMAN, C. A. (1979). Effect of naloxone on analgesia induced by food deprivation. Life Sciences, 25, 885-888.

MiNEKA, S., COOK, M., \& Miller, S. (1984). Fear conditioned with escapable and inescapable shock: Effects of a feedback stimulus. Journal of Experimental Psychology: Animal Behavior Processes, 10, 307-323.

Mineka, S., \& Hendersen, R. W. (1985). Controllability and predictability in acquired motivation. Annual Review of Psychology, 36, 495-529.

Prokasy, W. F. (1956). The acquisition of observing responses in the absence of differential external reinforcement. Journal of Comparative \& Physiological Psychology, 49, 131-135.

RODGERS, R. J., \& RANDALL, J. I. (1988). Environmentally induced analgesia: Situational factors, mechanisms and significance. In R. J. Rodgers \& S. J. Cooper (Eds.), Endorphins, opiates and behavioural processes (pp. 107-142). New York: Wiley.

Seligman, M. E. P. (1968). Chronic fear produced by unpredictable electric shock. Journal of Comparative \& Physiological Psychology, 66, 402-411.

Sonada, A., OKayasu, T., \& Hirai, H. (1991). Loss of controllability in appetitive situations interferes with subsequent learning in aversive situations. Animal Learning \& Behavior, 19, 270-275.

STROEBEL, C. (1969). Biologic rhythm correlates of disturbed behavior in the monkey. Bibliotheca Primatologica, 9, 91-105.

Tazi, A., Dantzer, R., \& Le Moal, M. (1987). Prediction and control of food rewards modulate endogenous pain inhibitory systems. Behavioral Brain Research, 23, 197-204.

Terman, G. W., Shavit, Y., Lewis, J. W., Cannon, J. T., \& Liebeskind, J. C. (1984). Intrinsic mechanisms of pain inhibition: Activation by stress. Science, 226, 1270-1276.

TOMIE, A., MuRPHY, A. L., \& FAITH, S. (1980). Retardation of autoshaping following pretraining with unpredictable food: Effects of changing the context between pretraining and testing. Learning \& Motivation, 11, 117-134.

Tsuda, A., Ida, Y., Satoh, H., Tsujimaru, S., \& Tanaka, M. (1989). Stressor predictability and rat brain noradrenaline metabolism. Pharmacology, Biochemistry \& Behavior, 32, 569-572.

Volpicelli, J. R., Ulm, R. R., \& AltenOR, A. (1984). Feedback during exposure to inescapable shocks and subsequent shock-escape performance. Learning \& Motivation, 15, 279-286.

Wasserman, E. A., \& Molina, E. J. (1975). Explicitly unpaired key light and food presentations: Interference with subsequent autoshaped key pecking in pigeons. Journal of Experimental Psychology: Animal Behavior Processes, 104, 30-38.

Watkins, L. R., Cobelli, D. A., Faris, P., ACEto, M. D., \& MaYer, D. J. (1982). Opiate vs. non-opiate footshock-induced analgesia (FSIA): The body region shocked is a critical factor. Brain Research, 242, 299-308.

WeIss, J. M. (1968). Effects of coping responses on stress. Journal of Comparative \& Physiological Psychology, 65, 251-260.

WEISs, J. M. (1970). Somatic effects of predictable and unpredictable shock. Psychosomatic Medicine, 32, 397-408.

WeIss, J. M. (1971). Effects of coping behavior in different warning signal conditions on stress pathology in rats. Journal of Comparative \& Physiological Psychology, 77, 1-13.

(Manuscript received January 5, 1994; revision accepted for publication May $25,1994$. 